\title{
A novel TP53 germline inframe deletion identified in a Spanish series of Li-fraumeni syndrome suspected families
}

\author{
Patricia Llovet $^{1} \cdot$ Francisco J. Illana $^{2} \cdot$ Lorena Martín-Morales $^{1} \cdot$ Miguel de la Hoya $^{1}$ • \\ Pilar Garre $^{1} \cdot$ M. Dolores Ibañez-Royo ${ }^{1}$ Pedro Pérez-Segura ${ }^{3} \cdot$ Trinidad Caldés $^{1}$. \\ Vanesa García-Barberán ${ }^{1}$
}

Published online: 1 June 2017

(C) Springer Science+Business Media Dordrecht 2017

\begin{abstract}
Li-Fraumeni syndrome (LFS) is an autosomal dominant, inherited tumor predisposition syndrome associated with heterozygous germline mutations in the TP53 gene. The molecular diagnosis of LFS is important to develop strategies for early detection and access to the genetic counseling. Our study evaluated germline TP53 mutations in Spanish families with a history suggestive of LFS. Germline TP53 alterations in 22 families with a history suggestive of LFS were evaluated by Sanger sequencing and multiplex ligation-dependent probe amplification. Loss of heterozygosity analysis and immunohistochemistry of the protein in the tumor were performed in order to evaluate the pathogenicity of a novel alteration detected. A total of seven TP53 mutations were detected, six point mutations (4 missense and 2 nonsense) and a novel inframe
\end{abstract}

Patricia Llovet and Francisco J. Illana have contributed equally to this study.

Electronic supplementary material The online version of this article (doi:10.1007/s10689-017-9990-0) contains supplementary material, which is available to authorized users.

Trinidad Caldés

trinidad.caldes@salud.madrid.org

$\triangle$ Vanesa García-Barberán

vanesa.garciabar@salud.madrid.org

1 Molecular Oncology Laboratory, Department of Medical Oncology, Instituto de Investigación Sanitaria San Carlos, IDISSC, CIBERONC, 28040 Madrid, Spain

2 Proteomic and Metabolomic Unit and Clinical Laboratory Department, Instituto de Investigación Sanitaria San Carlos (IdISSC), Madrid, Spain

3 Genetic Counseling Unit, Department of Medical Oncology, Hospital Universitario Clínico San Carlos, 28040 Madrid, Spain deletion. $93 \%$ of mutation carriers developed at least one malignancy (mainly breast cancer and sarcomas), with a mean age at diagnosis of the first tumor of 30.2 years. Two missense mutations acted as dominant-negative. The novel inframe mutation c.437_445del was located in the DNAbinding domain. This mutation segregated with cancer in the family, and both high expression of the protein and loss of the wild-type TP53 allele were detected in the tumor of the carrier. We have found a novel inframe deletion in TP53 that likely results in the loss of p53 function and acts in a non-dominant negative way, although further studies are necessary to clarify this issue. The identification of novel TP53 alterations is crucial for a personalized cancer-risk management of the Li-Fraumeni syndrome.

Keywords TP53 - Mutation · Li-Fraumeni syndrome · Dominant negative

\section{Introduction}

Li-Fraumeni syndrome (LFS) is a hereditary predisposition to cancer affecting adults and children that is caused by germline mutations in the TP53 tumor suppressor gene [1]. p53 is a DNA damage response protein, and its inactivation could be expected to result in subsequent genomic instability. As it is widely known, p53 is the major tumor suppressor protein that serves as a gatekeeper of cellular fate in multicellular organisms, and functions as a transcription factor of genes involved in the regulation of the cell cycle, DNA repair, apoptosis, cellular metabolism and senescence [2]. Between $50-70 \%$ of 'classic' LFS families carry a mutant TP53, and penetrance is nearly $100 \%$ by age 70 . Carriers of a germline TP53 mutation have a 50\% chance of developing cancer before the age of 40 , as compared with a 
$1 \%$ in the general population, and $90 \%$ of the carriers are diagnosed with cancer by the age of 60 years [1]. This gene predisposes to a wide spectrum of tumors, with an incidence rate of $50 \%$ for breast cancer, $15 \%$ for soft tissue sarcomas, $6 \%$ for brain tumors and 5\% for osteosarcomas [3]. Furthermore, other types of cancer, including lung, gastric, ovarian, colorectal cancer and early-onset melanoma, have also been described in excess in some families [1, 4]. In female TP53 mutation carriers, early-onset breast cancer is the most frequent malignancy, with an 18- to 60-fold increased risk when compared to the general population. In contrast, TP53 germline mutations only account for $4 \%$ of breast cancer families, with an average age at diagnosis of 30 years [5].

The diagnosis of classic LFS is based on an evolving set of clinical classification criteria that has been established using aspects of family history, type of tumors and early age at diagnosis, indicating when TP53 genotyping should be evaluated. However, TP53-positive families have been detected with incomplete clinical LFS features, leading to several definitions of Li-Fraumeni-like (LFL) syndrome. According to the NCCN guidelines, two criteria are considered to facilitate the identification of individuals for TP53 gene mutation testing: classic LFS and Chompret criteria (last updated in 2015) [6-8]. The molecular diagnosis of LFS is important in order to develop strategies for early detection, and it is significant for the clinical practice in a genetic counseling context. All patients should have cancer genetic counseling prior to initiating the testing process, and access to long-term counseling to support the educational and psychosocial needs of LFS patients and their families [4]. Screening protocols can detect early-stage disease and improve the outcome in LFS patients [4, 9]. Recently, a study has showed that a surveillance protocol for TP53 mutation carriers is associated with early tumor detection and improves long-term survival $[10,11]$. In a study analyzing the largest worldwide series of patients suggestive of LFS, Bougeard et al. pointed out the contribution of chemotherapy and radiotherapy to the increase of secondary tumor risk. Moreover, they observed a clinical severity gradient of germline TP53 mutations, suggesting that the clinical management of the carriers might be stratified according to the class of the mutation, although this should be confirmed in subsequent studies [8].

The molecular study of LFS has been focusing on the genetic screening of exons 5-8 of TP53, where $95 \%$ of the mutations are detected [12], although other mutations can occur outside this region. Currently, the molecular study is performed by sequencing the entire coding region. DNA sequencing and immunohistochemical staining have been used to assess TP53 status. Most TP53 alterations are point missense mutations that lead to the synthesis of a stable but inactive protein that accumulates in the nucleus of tumor cells, enabling p53 detection with routine IHC. For this reason, immunohistochemistry (IHC) is commonly used to evaluate mutant p53 status: overexpression of p53 is associated with a mutation, and the lack of expression with wild-type p53. However, the correlation between the accumulation of p53 and TP53 mutations is about $80 \%$, since frameshift mutations do not lead to p53 accumulation [9].

In this study, we included Spanish cancer families that met either the criteria for classic LFS or Chompret criteria. Seven pathogenic variants were detected in our population, one of which had never been reported in the literature.

\section{Materials and methods}

\section{Study population}

Forty five members from 22 families with a history suggestive of LFS and recruited at the Genetic Counseling Unit of our Hospital (2000-2015) were analyzed. This study was revised and approved by the Ethics Committee of the Clínico San Carlos Hospital of Madrid and was conducted in accordance with the Declaration of Helsinki. All members of the families who contributed to this study or their legal representatives gave their informed consent. Families were classified into two groups: classic LFS and Chompret criteria (updated by Bougeard et al.) [8]. Classic LFS criteria included families with a proband diagnosed of a sarcoma before 45 years of age, a first-degree relative with a cancer diagnosed at age $<45$ years, and a first-degree or second-degree relative presenting any cancer with age of onset $<45$ or a sarcoma at any age. Chompret criteria included probands with a tumor belonging to the LFS tumor spectrum (e.g. sarcoma, CNS tumor, premenopausal breast cancer, ACC) before age 46 AND at least one firstor second-degree relative with a LFS tumor (except breast cancer if the proband had breast cancer) before 56 years old OR with multiple tumors; OR a proband with multiple primary tumors (except multiple breast tumors), two of which belonged to the LFS tumor spectrum and the first occurring before age 46 years; OR a patient with ACC, choroid plexus tumor, or rhabdomyosarcoma of embryonal anaplastic subtype, irrespective of the family history; OR breast cancer before age 31 years. TP53 mutation testing was offered to family members of a proven TP53 mutation carrier. Clinical data were updated until 2015 for each TP53 mutation carrier. The histological subtypes of cancer were confirmed in the medical and pathology records. The sequence of the TP53 gene was also analyzed in a group of families that did not fulfill the previous criteria, but that included at least a sarcoma at any age. In this group no alterations were found, in agreement with another study [13]. This seems to 
indicate that TP53 testing should not be recommended in this type of families.

\section{DNA extraction}

Germline DNA was isolated from peripheral blood lymphocytes by the salting out procedure [14]. Tumor DNA extraction was performed using the "QIAamp DNA FFPE Tissue" Kit (Qiagen), following the manufacturer's instructions. The tumor tissue was microdissected on a corresponding unstained slide for subsequent DNA isolation. Five serial 5-7 $\mu \mathrm{m}$-thick sections were cut from tumor paraffin blocks.

DNA quantity and quality were assessed by Nanodrop (ND1000), and all samples were diluted to a final concentration of $50 \mathrm{ng} / \mu \mathrm{l}$.

\section{Immunohistochemistry (IHC)}

Tumor tissue sections of paraffin-embedded specimens were selected for p53 IHC staining using a recombinant human wild-type p53 antibody (DO-7; Dako). After the deparaffinization and hydration of the sections, antigens were unmasked by heat in EDTA buffer. Immunostaining was performed using the UltraVision LP Large Volume Detection System AP Polymer (Thermo Scientific, Waltham, MA, USA). Negative control slides were included. Nuclear staining was considered a positive reaction. The extent of the staining was estimated to the nearest $10 \%$ level of positive tumor cells. The slides were reevaluated by two pathologists. Each section was scanned at $\times 100$ and $\times 400$ magnification by microscope (Olympus BX51).

\section{TP53 gene sequencing}

Exons 2-11 and the flanking regions of the TP53 gene were amplified by PCR using specific primers with $100 \mathrm{ng}$ of DNA. PCR primers had a common sequence to unify the sequencing process, which used the same forward and reverse primers for all exons (primers are summarized in Supplementary Table 1). The extended PCR products were purified and sequenced using the ABI3130 automated sequencer (Applied Biosystems, USA). Sequencing was carried out using the fluorescent Big-Dye Terminator v.1.1 cycle sequencing Kit (Applied Biosystems, Foster City, CA, USA) according to the manufacturer's protocol. The NCBI reference genomic sequence used for TP53 was NC_000017.10.

\section{Mutilplex ligation-dependent probe amplification (MLPA)}

Large deletions or genomic rearrangements of patients were analyzed by a commercial MLPA kit (SALSA MLPA probemix P056-B1 TP53, MRC Holland) according to the manufacturer's protocols. DNA (100 ng) extracted from peripheral blood was used. MLPA PCR products were separated on the ABI3130 instrument (Applied Biosystems) and peak heights for each PCR product were compared to a normal sample using the Coffalyser software to determine gene dosage for each individual exon.

\section{Analysis of loss of heterozygosity (LOH) at the 17q11.2 locus}

The microsatellite marker D17S250 was used to determine the $\mathrm{LOH}$ at the 17q11.2 locus using a fluorescent PCR in both germline and tumor DNA. Products were analyzed in the ABI310 genetic analyzer and the area ratio between tumor DNA and wild-type DNA was calculated to determine the allelic loss. Area ratios below 0.5 were considered LOH. Loss of heterozygosity was also studied through DNA tumor sequencing. Details of the primers are summarized in Supplementary Table 2.

\section{Data analysis and in silico predictions}

The effects of TP53 alterations on the protein structure and function were identified using ClinVar and the IARC TP53 Database R18 (April 2016). MutationTaster was used to predict the possible impact of DNA changes in TP53 with default settings. The Rasmol (v2.7.2) program was used for the visualization of p53's 3D structure and the evaluation of the impact of an alteration on the p53 protein. This analysis was based on conformational parameters for amino acids in helixes, $\beta$-sheets and random coil regions from proteins [15]. The effect of mutations on splicing was predicted using Human Splicing Finder [16]. The frequencies in control cohorts were examined in individuals from the Exome Variant Server (Exome Variant Server, NHLBI GO Exome Sequencing Project (ESP), Seattle, WA (URL: http://evs. gs.washington.edu/EVS/) [date (April, 2016) accessed]) and ExAC (Exome Aggregation Consortium (ExAC), Cambridge, MA (URL: http://exac.broadinstitute.org) [date (April, 2016) accessed]). The reference sequences used were: GenBank NC_000017.10 (genomic), NM_000546.5 (cDNA) and UniProt P04637 (protein). 


\section{Results}

\section{Findings in our families}

The germline mutational status of the entire coding region of TP53 was analyzed in 22 families (45 members) with history suggestive of LFS. Among these, five families fulfilled the classic LFS criteria and 17 families fulfilled the Chompret criteria. In addition, all classic LFS families also fulfilled the Chompret criteria. TP53 mutations were detected in a total of seven families: four LFS and three Chompret families. Table 1 shows clinical and molecular data from the studied members. Large deletions and insertions analyzed by MLPA were not detected. Twenty-six members of the TP53-positive families were studied, 14 of whom were mutation carriers (10 in LFS and 4 in Chompret families). The mutation detection rate was $80 \%$ for LFS, and $18 \%$ for Chompret criteria.

The mean age at tumor diagnosis was 35.1 years, the first tumor in mutation carriers being diagnosed at a mean age of 30.2 years. The age of tumor onset was more homogeneous in females than in males (Fig. 1a, b). Thirteen TP53 mutation carriers (93\%) developed at least one malignancy (9 females and 4 males), with a total of 18 tumors. Multiple primary tumors were diagnosed in $30.1 \%$ of the carriers. Tumor distribution in the mutation carriers is shown in Fig. 2. Sarcomas were the most frequent tumors in males $(60 \%)$, whereas females were mainly affected of breast cancer $(54 \%$, being the first tumor in $78 \%$ of the cases).

Table 1 Clinical characteristics and TP53 status only of the members analyzed from the TP53-positive families

\begin{tabular}{|c|c|c|c|c|c|c|}
\hline Family ID & Criteria & Gender & Family member & $\begin{array}{l}\text { Tumor type-age } \\
\text { of onset }\end{array}$ & TP53 status & TP53 IHC in tumor \\
\hline \multirow[t]{5}{*}{4} & \multirow[t]{5}{*}{ Chompret } & $\mathrm{F}$ & Cancer & B-26 & c. $1015 \mathrm{G}>\mathrm{C}$ & positive \\
\hline & & $\mathrm{F}$ & Asymptomatic & - & wt & NA \\
\hline & & M & Asymptomatic & - & wt & NA \\
\hline & & $\mathrm{F}$ & Asymptomatic & - & $\mathrm{wt}$ & NA \\
\hline & & $\mathrm{F}$ & Asymptomatic & - & wt & NA \\
\hline 8 & Chompret & M & Cancer & Cp-4 & c. $743 \mathrm{G}>\mathrm{A}$ & ND \\
\hline \multirow[t]{2}{*}{12} & \multirow[t]{2}{*}{ Chompret } & $\mathrm{F}$ & Cancer & OS-23 & c. $.586 \mathrm{C}>\mathrm{T}$ & Positive \\
\hline & & M & Asymptomatic** & - & c. $.586 \mathrm{C}>\mathrm{T}$ & NA \\
\hline \multirow[t]{3}{*}{15} & \multirow[t]{3}{*}{ LFS and Chompret } & $\mathrm{F}$ & Cancer & B-34/STS-36/O-39 & c. $916 \mathrm{C}>\mathrm{T}$ & Positive \\
\hline & & M & Cancer & STS-37 & c. $916 \mathrm{C}>\mathrm{T}$ & Positive \\
\hline & & $\mathrm{F}$ & Asymptomatic & - & wt & NA \\
\hline \multirow[t]{5}{*}{29} & \multirow[t]{5}{*}{ LFS and Chompret } & $\mathrm{F}$ & Cancer & B-31/STS-43 & c. $799 \mathrm{C}>\mathrm{T}$ & Positive \\
\hline & & $\mathrm{F}$ & Cancer & B-34 & c. $799 \mathrm{C}>\mathrm{T}$ & Positive \\
\hline & & $\mathrm{F}$ & Cancer & $\mathrm{Ne}-27$ & wt & ND \\
\hline & & $\mathrm{F}$ & Asymptomatic & - & wt & NA \\
\hline & & $\mathrm{F}$ & Asymptomatic & - & wt & NA \\
\hline \multirow[t]{7}{*}{30} & \multirow[t]{7}{*}{ LFS and Chompret } & $\mathbf{F}$ & Cancer & STS-28 & c.437_445del & Positive \\
\hline & & $\mathrm{F}$ & Cancer & B2-45/O-49 & c.437_445del & Positive \\
\hline & & $\mathrm{F}$ & Cancer & B-30 & c.437_445del & Positive \\
\hline & & $\mathrm{F}$ & Cancer & B-30 & c.437_445del & Positive \\
\hline & & M & Cancer & Me-58/STS-58 & c.437_445del & Positive \\
\hline & & $\mathrm{F}$ & Asymptomatic & - & wt & NA \\
\hline & & M & Asymptomatic** & - & c. $437 \_445 \mathrm{del}$ & NA \\
\hline \multirow[t]{4}{*}{32} & \multirow[t]{4}{*}{ LFS and Chompret } & M & Cancer & STS-12 & c. $733 \mathrm{G}>\mathrm{A}$ & Positive \\
\hline & & $\mathrm{F}$ & Cancer & B-32 & wt & ND \\
\hline & & $\mathrm{F}$ & Asymptomatic & - & wt & NA \\
\hline & & $\mathrm{F}$ & Asymptomatic & - & wt & NA \\
\hline
\end{tabular}

The NCBI reference genomic sequence used for TP53 was NM_000546.5

LFS Classical Li-Fraumeni syndrome criteria, Chompret chompret criteria revised in $2015, F$ female, $M$ male, $B$ breast cancer, $B 2$ bilateral breast cancer, $M e$ Melanoma, Ne neuroendocrine tumor, STS soft tissue sarcoma, $O$ Ovarian cancer, $O S$ Osteosarcoma, $N A$ not applicable, $N D$ IHC not done

**Young asymptomatic mutation carrier (27 years-old and 5 years-old in families 12 and 30, respectively). The member of the family with the malignant Phyllodes tumor is shown in bold 
Fig. 1 a Age at tumor diagnosis, and $\mathbf{b}$ age of first tumor onset in TP53 mutation carriers in females and males
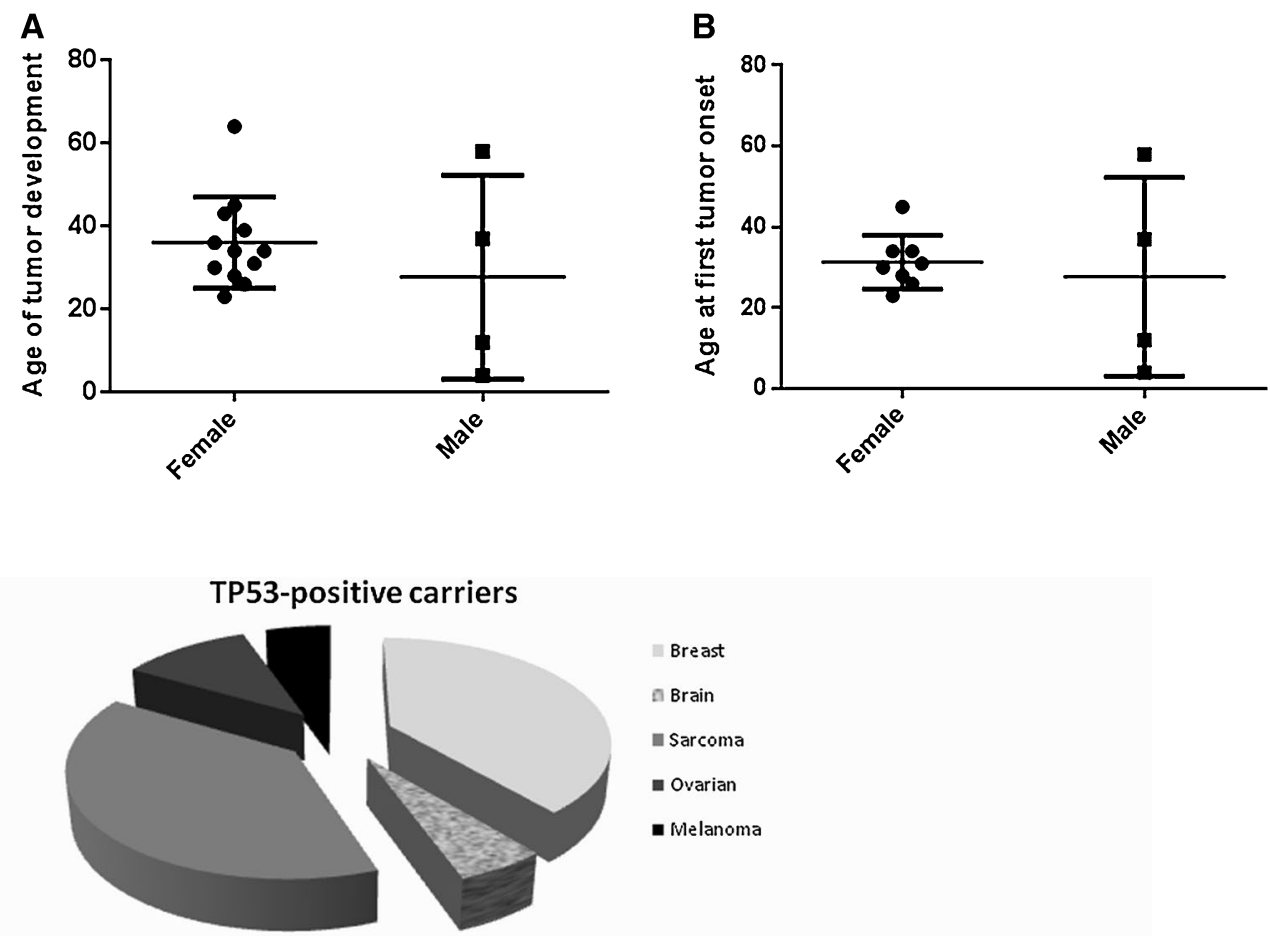
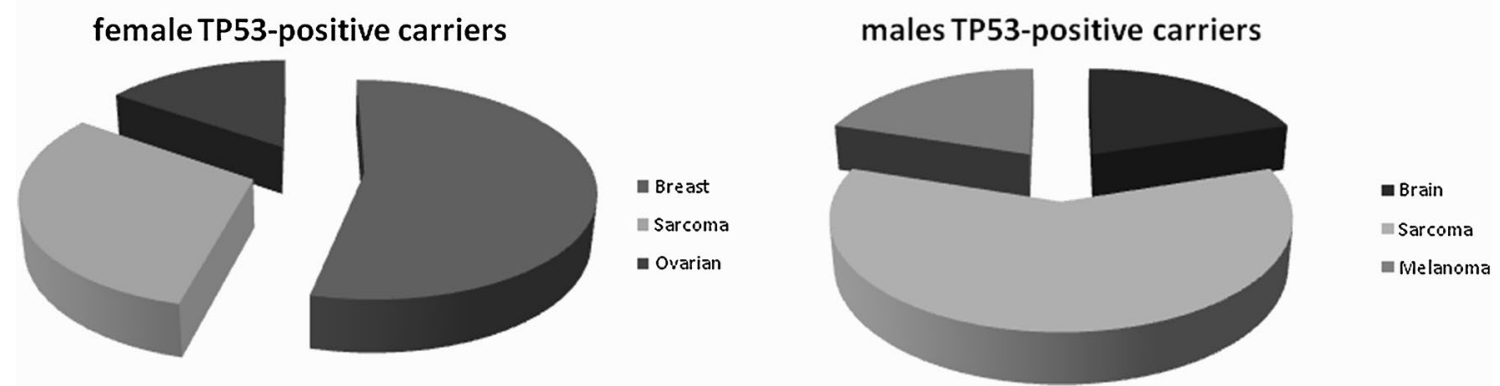

Fig. 2 Tumor distribution of TP53 mutation carriers in the total series, females and males

\section{Germline alterations detected in the TP53 gene}

Seven germline TP53 alterations were detected in the index cases from seven different TP53-positive families. Additional information about the family histories is shown in Supplementary Table 3 . The alterations detected corresponded to six point mutations (4 missense and 2 nonsense) and a novel inframe deletion. The sequence of the inframe deletion is shown in Fig. 3 ( $\mathrm{a}$ and $\mathrm{b}$ panels). Among the seven germline mutations found, five affected the DNAbinding domain, one affected the tetramerization domain and one affected the C-terminal domain. Family members with missense mutations detected in the p53 DNAcontact residues (exon 7) showed an early tumor onset, developing malignances at ages of four (choroid plexus tumor; c.743G $>$ A) and 12 years (sarcoma; c. 733G $>$ A). Details about the germline TP53 alterations identified were obtained using ClinVar and the IARC TP53 Database, with the exception of the inframe deletion that had not been reported in these databases (Table 2). In addition, the deleterious effect on the protein function and structure was predicted for all detected mutations by the MutationTaster program. Splice sites were not affected by the mutations (Human Splicing Finder program). Finally, Supplementary Fig. 1 shows how the different p53 protein isoforms are affected by each alteration. In control cohorts from the ExAc database (non Finnish European) and from the Exome Variant Server (European American), our identified mutations showed minor allele frequencies of $<0.01 \%$.

\section{Novel TP53 mutation detected in LFS family ID30}

Family ID30 was compatible with Hereditary Breast and Ovarian Cancer Syndrome, and so the BRCA1/BRCA2 
A Blood: Wt
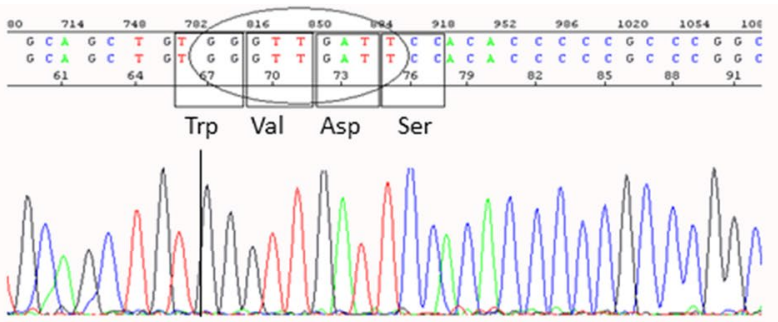

B Blood: c.437de19; p.Trp146_Asp148del

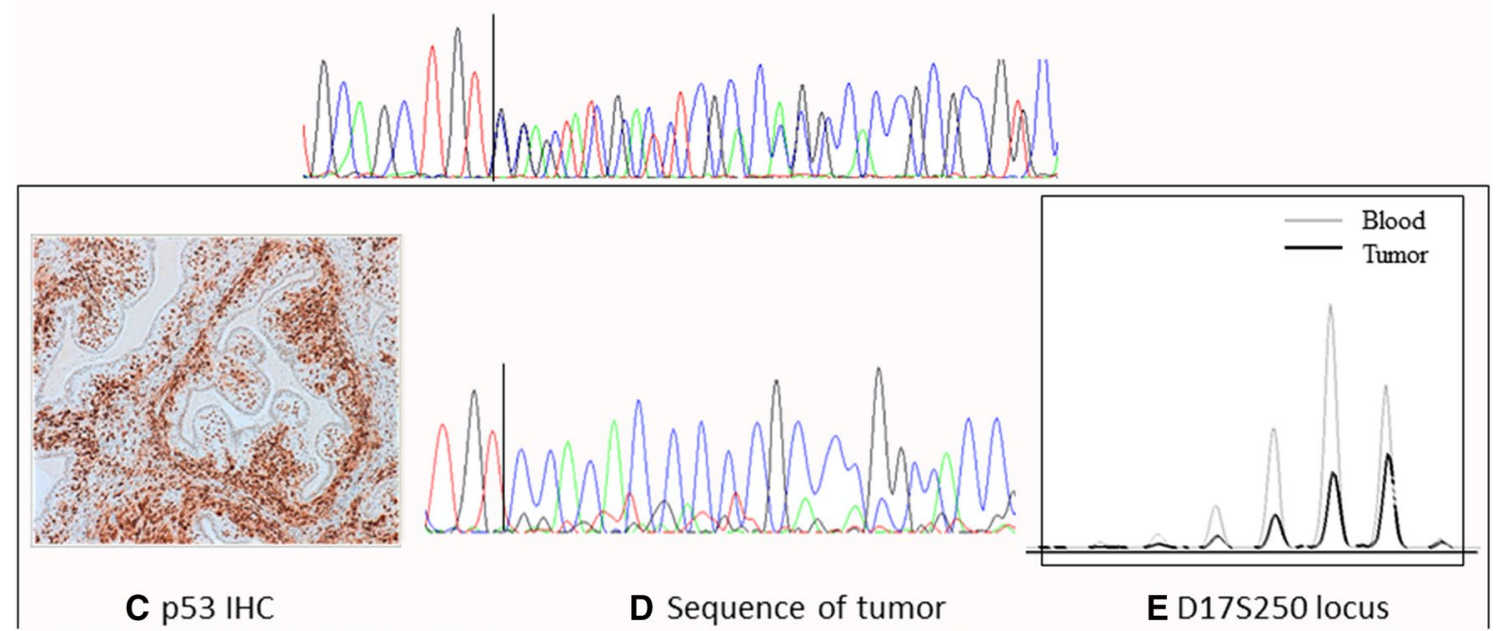

Fig. 3 Study of the inframe deletion c.437 445del in exon 5 of TP53 found in our ID30 family. Panel $a$ and $\bar{b}$ wild-type and mutant sequences. Panel $c$ staining of the Phyllodes tumor with the anti-p53
DO-7. Neoplastic tissue shows strong nuclear staining. Panels $d$ and $e$ $\mathrm{LOH}$ of one allele is shown by the sequence and the D17S250 microsatellite study in the tumor

Table 2 Molecular data of the TP53 alterations detected in our families

\begin{tabular}{|c|c|c|c|c|c|c|c|}
\hline $\begin{array}{l}\text { Nucleotide muta- } \\
\text { tion }\end{array}$ & Exon & Protein & Effect & Domain function & Structural motif & $\begin{array}{l}\text { Clinical signifi- } \\
\text { cance (ClinVar) }\end{array}$ & $\begin{array}{l}\text { Mutationtaster } \\
\text { prediction }\end{array}$ \\
\hline c. $437 \_445 \mathrm{del}$ & 5 & $\begin{array}{l}\text { p.(W146_ } \\
\text { D148del) }\end{array}$ & Inframe deletion & DNA binding & NDBL/beta-sheet & NA & Disease causing \\
\hline c. $586 \mathrm{C}>\mathrm{T}$ & 6 & p.R196* & Nonsense & DNA binding & NDBL/beta-sheet & Pathogenic & Disease causing \\
\hline c. $733 \mathrm{G}>\mathrm{A}$ & 7 & p.G245S & Missense & DNA binding & L2/L3 & Pathogenic & Disease causing \\
\hline c. $743 \mathrm{G}>\mathrm{A}$ & 7 & p.R248Q & Missense & DNA binding & L2/L3 & Pathogenic & Disease causing \\
\hline c. $799 \mathrm{C}>\mathrm{T}$ & 8 & p.R267W & Missense & DNA binding & NDBL/beta-sheet & Likely pathogenic & Disease causing \\
\hline c. $916 \mathrm{C}>\mathrm{T}$ & 8 & p.R306* & Nonsense & NA & C-term & Pathogenic & Disease causing \\
\hline c. $1015 \mathrm{G}>\mathrm{C}$ & 10 & p.E339Q & Missense & tetramerization & $\begin{array}{l}\text { C-term/tetrameri- } \\
\text { zation }\end{array}$ & $\begin{array}{l}\text { Uncertain signifi- } \\
\text { cance }\end{array}$ & Disease causing \\
\hline
\end{tabular}

The NCBI reference genomic sequence used for TP53 was NM_000546.5. NA Not applicable, $N D B L$ non-DNA-binding loop, $L$ loop

*Stop codon in the protein as HGVS rules

genes were studied in germline DNA. However, germline mutations and large rearrangements—analyzed according to previous studies $[17,18]$ - were not found for either gene (data not shown and available upon request). Some years later, a 28 -year-old member was diagnosed of a malignant Phyllodes tumor of the breast, and the study of the TP53 gene was thus proposed. High levels of the p53 protein were detected in tumor tissue using an IHC analysis (Fig. 3c). Sequencing of the TP53 gene was therefore performed in germline DNA, showing a deletion of nine nucleotides in exon 5 (c.437_445del; p.(Trp146_ Asp148del)). This deletion produced the lack of three amino acids, though maintaining the open reading frame (Fig. 3a, b). 
Moreover, the loss of heterozygosity was analyzed through TP53 sequencing in DNA from microdissected tumor cells and through microsatellite study (Fig. 3d, e). The 17S250 locus (17q11.2) was used to estimate the LOH between the blood sample and the tumor sample $(\mathrm{R}=0.5)$. Both studies suggested a loss of the wild-type allele.

The in silico analysis by MutationTaster predicted this mutation to be disease causing. The deletion included the end of a beta sheet (which consists of six amino acids, from Cys141 to Trp146) and the surrounding region (Supplementary Fig. 2a). An in silico analysis with Rasmol was performed in order to evaluate the impact of this deletion on the protein structure. The deletion was located in the DNA-binding domain, but the amino acids involved did not seem to have a direct interaction with the DNA (Supplementary Fig. 2b). The predictive factors for the secondary structure of the mutant protein suggested a stabilization of the beta sheet, while the surrounding aperiodic structure would comprise two residues less. In this sense, important changes in the 3D structure of the mutant protein were not predicted.

On the other hand, a segregation study of this variant within the ID30 family was performed. The variant cosegregated with cancer in the family (Fig. 4); the deletion was detected in a woman with malignant Phyllodes tumor at 28 years old, in a deceased woman with bilateral breast cancer and ovarian cancer at 45 and 49 years respectively, in two women with breast cancer at 30 years, in a man with melanoma and sarcoma at 58 years and in an asymptomatic 5 -year-old child. Moreover, the alteration was not detected in a healthy 49 years woman.

\section{Discussion}

Germline TP53 mutations have been observed in $50-70 \%$ of the families with Li-Fraumeni syndrome, a dominant inherited cancer syndrome with a high rate of early-onset

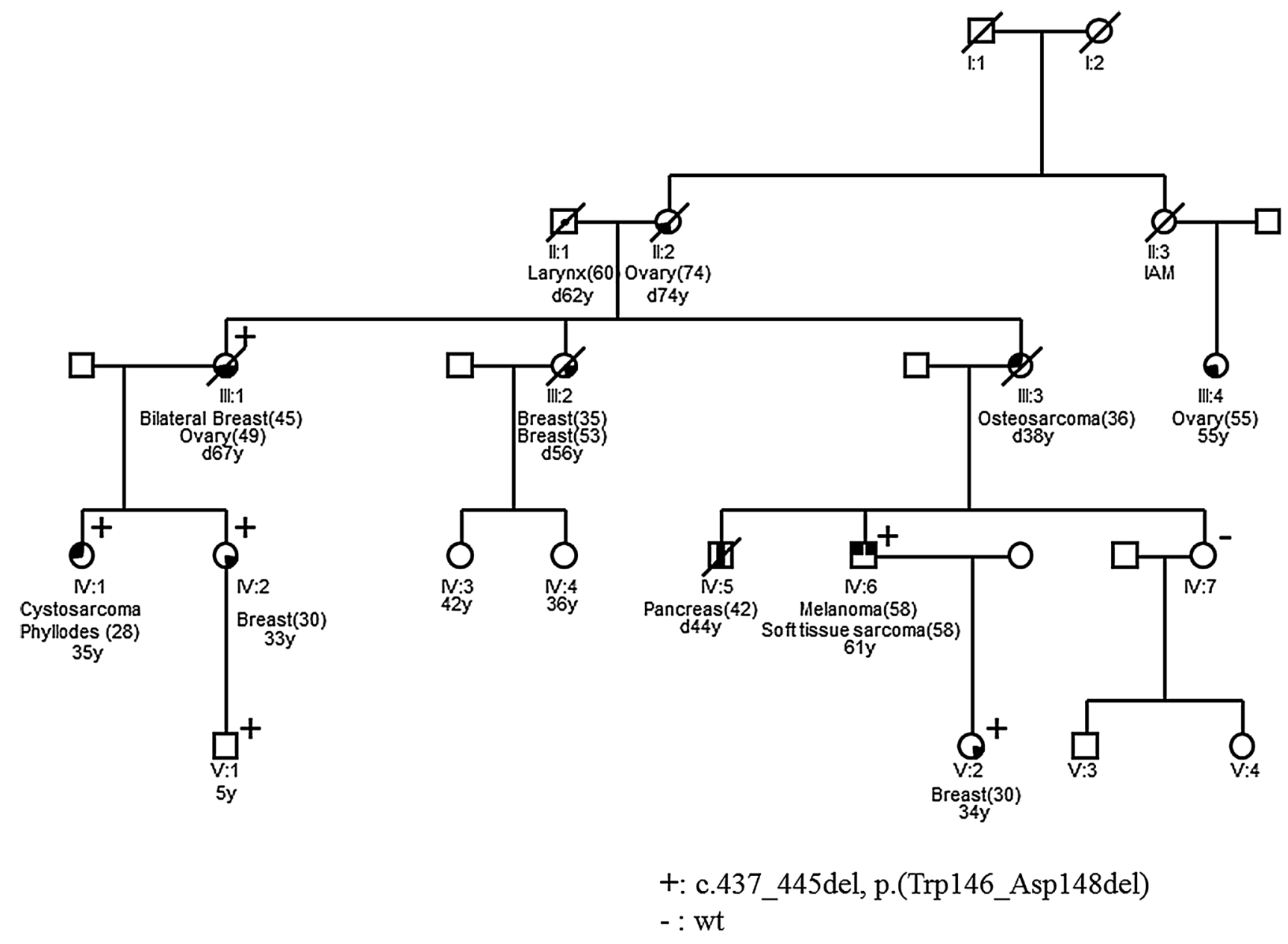

Fig. 4 Pedigree of the family with the novel alteration identified (c.437_445del, p.(Trp146_Asp148del)). The different cancer types are indicated with different black colored sections. The result of the

genetic study of TP53 (+: presence of deletion/-: absence of deletion), age at diagnosis (brackets) and age at time of the last clinical revision $(y)$ or death $(d-y)$ are also shown 
breast cancer as well as other multiple tumor types (6). In this work, we have investigated germline TP53 mutations in 22 Spanish cancer families that met either the criteria for classic LFS or Chompret criteria (updated in 2015). By doing so, seven germline pathogenic variants were identified. In our population, the highest TP53 mutation rate was detected in individuals who fulfilled the LFS criteria.

In the mutation carriers from our study, the tumor spectrum and age of onset were in line with previous studies [4]. The most frequent tumors in TP53 mutation carriers were breast cancers and sarcomas. Breast cancers were diagnosed at an early age (26-34 years), except for one case with a bilateral breast cancer at 45 years. In $71 \%$ of the sarcomas, the age of distribution was between ages 20 and 40, which is according with a previous study analyzing germline TP53 mutations in an adult-onset sarcoma cohort [19].

As far as the germline mutations are concerned, seven pathogenic variants were detected, including four missense mutations (c.733G $>$ A, c.743G $>$ A, c.799C $>$ T and c. $1015 \mathrm{G}>\mathrm{C}$ ), two nonsense mutations (c.586C $>\mathrm{T}$ and c.916C > T) and a novel inframe deletion (c.437_445del) not reported previously. TP53 encodes different p53 protein isoforms, resulting from alternative splicing, alternative promoter usage, and alternative initiation of translation. N-terminal and C-terminal p53 isoforms can inhibit or enhance the canonical p53 activity, respectively, and are often deregulated in cancer [20]. The mutations identified in our study affect the majority of p53 isoforms, as do other mutations within exons 5-8 described in the literature, which represent $95 \%$ of the TP53 alterations. Nevertheless, more studies are necessary regarding the effects that mutations affecting different isoforms have on the p53 pathway.

The alteration in exon 5 (c.437_445del; p.(Trp147_ Asp148del)) had not been reported in the literature and involved the loss of three amino acids in the DNA-binding domain of the p53 protein, but did not seem to be crucial to bind specific DNA. In order to evaluate the pathogenicity of this mutation, in silico studies were performed. MutationTaster considered this variant as pathogenic, while the 3D analysis did not clarify this point. Immunohistochemistry in the malignant Phyllodes tumor showed a high expression of the protein similar to that seen in patients with pathogenic point missense mutations, due to the accumulation of p53. Bearing in mind that the mutation cosegregated with cancer in different family members, our results suggest that the inframe deletion shows a concordance with a pathogenic mutation.

Most tumor suppressors require the loss of both alleles for tumor development, but p53 hotspot mutations in just one allele result in severe loss of function. Mutations described in TP53 are mostly missense and may act in a dominant-negative fashion, blocking the normal wild-type functions. Thus, transgenic mice expressing mutated p53 have higher incidence of tumor formation compared to p53+/- mice [21]. Notably, LFS patients with mutations in the p53 DNA-contact residues have a decreased rate of loss of heterozygosity, suggesting that the missense mutations in one allele that make p53 incapable of binding specific DNA are sufficient to lose the function of the remaining wild-type p53. The p53 tetramer binds to its specific DNA using both of its dimers, which means that one mutant dimer present in the tetramer would be enough to abolish the binding and to impair the transcription activation function of p53 [22].

Importantly, families carrying missense mutations within the DNA-binding domain of TP53 show a higher penetrant cancer phenotype than families with proteintruncating or protein-inactivating mutations [8]. Indeed, it has been reported that dominant-negative mutations constitute the most severe mutations among missense mutations and they are associated with an earlier tumor onset. In our series, two missense mutations (c.733G $>\mathrm{A}$ and c. $743 \mathrm{G}>\mathrm{A}$ ) in the core domain were classified as dominant-negative. These cases showed a high clinical severity, with an age of tumor onset of 4 and 12 years. The less severe alterations correspond to loss of function mutations that include nonsense mutations, frameshift mutations or genomic rearrangements, and they are usually associated with later tumor onset [8]. In this case, we showed a short inframe deletion, so we had to describe the phenotypic expression in this family in order to classify it as a severe or mild mutation.

As it is mentioned above, the novel inframe deletion was located in the DNA-binding domain. However, this deletion did not affect the residues responsible for the direct contact with DNA. The DNA sequencing and microsatellite study of p53 in the tumor of a family member suggested $\mathrm{LOH}$ of the TP53 wild-type allele. The LOH in the tumor suggests that the deletion may not act in a dominant-negative way, since the loss of the wild-type allele was necessary for tumor development. In conclusion, based on variant segregation, immunohistochemistry, loss of heterozygosity, in silico studies and late tumor onset in this family, this mutation may be considered as a pathogenic variant that acts in a non-dominant negative way.

The higher penetrance and earlier tumor onset associated with dominant-negative TP53 mutations emphasizes the importance of defining novel TP53 mutations. We agree with Bougeard and col. on the suggestion of stratifying the clinical management of TP53 carriers according to the class of their mutation [8]. Indeed, risk assessment in families attending the genetic counseling, as well as possible predictive testing, must take into account the type of mutation. The severe dominant-negative missense mutations may constitute an argument for presymptomatic 
testing in children and annual screening protocols. However, in this case presymptomatic tests are only justified in the adults from this family.

Acknowledgements This work was supported by the Instituto de Salud Carlos III: ISCIII-RTICC-RD2012/0036/0006, CB-161200301 (Plan estatal de I+D + I 2013-2016, FEDER funds co-financed). We would like to thank Dr. José G. Gavilanes Franco (Complutense University of Madrid, Spain) for his assistance with the in silico characterization of the inframe deletion in $\mathrm{p} 53$. The authors also wish to thank the donors and the "Instituto de Investigación Sanitaria San Carlos" Biobank for the human specimens used in this study.

\section{Compliance with ethical standards}

Conflict of interest We state no conflicts of interest. The funding bodies had no role in the study design, data collection and analyses, decision to publish or preparation of the manuscript.

Informed consent Informed consents were obtained from all individual participants included in the study.

Research involving human participants All procedures performed in studies involving human participants were in accordance with the ethical standards of the institutional and/or national research committee and with the 1964 Helsinki declaration and its later amendments or comparable ethical standards.

\section{References}

1. Kamihara J, Rana HQ, Garber JE (2014) Germline TP53 mutations and the changing landscape of Li-Fraumeni syndrome. Hum Mutat 35:654-662. doi:10.1002/humu.22559

2. Vousden KH, Prives C (2009) Blinded by the Light: the growing complexity of p53. Cell 137:413-431. doi:10.1016/j. cell.2009.04.037

3. Mai PL, Best AF, Peters JA et al (2016) Risks of first and subsequent cancers among TP53 mutation carriers in the National Cancer Institute Li-Fraumeni syndrome cohort. Cancer 122:3673-3681. doi:10.1002/cncr.30248

4. Sorrell AD, Espenschied CR, Culver JO, Weitzel JN (2013) Tumor protein p53 (TP53) testing and Li-Fraumeni syndrome $\dagger^{-}$: current status of clinical applications and future directions. Mol Diagn Ther 17:31-47. doi:10.1007/s40291-013-0020-0

5. Lalloo F, Varley J, Ellis D et al (2003) Prediction of pathogenic mutations in patients with early-onset breast cancer by family history. Lancet 361:1101-1102. doi:10.1016/ S0140-6736(03)12856-5

6. Chompret A, Abel A, Stoppa-Lyonnet D et al (2001) Sensitivity and predictive value of criteria for p53 germline mutation screening. J Med Genet 38:43-47

7. Tinat J, Bougeard G, Baert-Desurmont S, et al (2009) 2009 version of the Chompret criteria for Li Fraumeni syndrome. J Clin Oncol 27:e108-e109. doi:10.1200/JCO.2009.22.7967
8. Bougeard G, Renaux-Petel M, Flaman J-M et al (2015) Revisiting Li-Fraumeni syndrome from TP53 mutation carriers. J Clin Oncol 33:2345-2352. doi:10.1200/JCO.2014.59.5728

9. Nguyen TA, Menendez D, Resnick MA, Anderson CW (2014) Mutant TP53 posttranslational modifications: challenges and opportunities. Hum Mutat 35:738-755. doi:10.1002/humu.22506

10. Villani A, Tabori U, Schiffman J et al (2011) Biochemical and imaging surveillance in germline TP53 mutation carriers with Li-Fraumeni syndrome: a prospective observational study. Lancet Oncol 12:559-567. doi:10.1016/S1470-2045(11)70119-X

11. Villani A, Shore A, Wasserman JD et al (2016) Biochemical and imaging surveillance in germline TP53 mutation carriers with Li-Fraumeni syndrome: 11 year follow-up of a prospective observational study. Lancet Oncol 17:1295-1305. doi:10.1016/ S1470-2045(16)30249-2

12. Palmero EI, Achatz MI, Ashton-Prolla P et al (2010) Tumor protein 53 mutations and inherited cancer: beyond Li-Fraumeni syndrome. Curr Opin Oncol 22:64-69. doi:10.1097/ CCO.0b013e328333bf00

13. Ruijs MWG, Verhoef S, Rookus MA et al (2010) TP53 germline mutation testing in 180 families suspected of Li-Fraumeni syndrome: mutation detection rate and relative frequency of cancers in different familial phenotypes. J Med Genet 47:421-428. doi:10.1136/jmg.2009.073429

14. Miller SA, Dykes DD, Polesky HF (1988) A simple salting out procedure for extracting DNA from human nucleated cells. Nucleic Acids Res 16:1215

15. Chou PY, Fasman GD (1974) Conformational parameters for amino acids in helical, beta-sheet, and random coil regions calculated from proteins. Biochemistry 13:211-222

16. Desmet F-O, Hamroun D, Lalande M et al (2009) Human splicing finder: an online bioinformatics tool to predict splicing signals. Nucleic Acids Res 37:e67. doi:10.1093/nar/gkp215

17. de la Hoya M, Gutiérrez-Enríquez S, Velasco E et al (2006) Genomic rearrangements at the BRCA1 locus in Spanish families with breast/ovarian cancer. Clin Chem 52:1480-1485. doi:10.1373/clinchem.2006.070110

18. Gutiérrez-Enríquez S, de la Hoya M, Martínez-Bouzas C et al (2007) Screening for large rearrangements of the BRCA2 gene in Spanish families with breast/ovarian cancer. Breast Cancer Res Treat 103:103-107. doi:10.1007/s10549-006-9376-8

19. Mitchell G, Ballinger ML, Wong S, et al (2013) High frequency of germline TP53 mutations in a prospective adult-onset sarcoma cohort. PloS ONE 8:e69026. doi:10.1371/journal.pone.0069026

20. Surget S, Khoury MP, Bourdon J-C (2013) Uncovering the role of p53 splice variants in human malignancy: a clinical perspective. OncoTargets Ther 7:57-68. doi:10.2147/OTT.S53876

21. Petitjean A, Achatz MIW, Borresen-Dale AL et al (2007) TP53 mutations in human cancers: functional selection and impact on cancer prognosis and outcomes. Oncogene 26:2157-2165. doi:10.1038/sj.onc. 1210302

22. Aramayo R, Sherman MB, Brownless K et al (2011) Quaternary structure of the specific p53-DNA complex reveals the mechanism of p53 mutant dominance. Nucleic Acids Res 39:89608971. doi:10.1093/nar/gkr386 